\title{
Research on a novel PLL based islanding detection technology for multi grid-connected inverters
}

\author{
Dong Xie , Dajin Zhang and Peng Gao \\ College of Electrical Engineering, Tongling University, Tongling, China \\ XDY@tlu.edu.cn, zdajin@163.com, tlgaopeng@qq.com
}

\begin{abstract}
Keywords: islanding detection; dilution effect; composite; phase-locked loop
Abstract. Aiming at the problem that under the condition of multi grid-connection inverters, the dilution effect can easily cause islanding detection failure of Sandia Frequency Shift (SFS) method, a new phase locked loop based composite islanding detection strategy for multi grid-connected inverters is proposed. The proposed strategy combines Sandia Voltage Shift (SVS) and SFS islanding detection method to improve the success rate of multi grid-connection inverters islanding detection. In addition, the SVS method is put into use only when the condition is met, which can avoid its adverse effects on the system energy efficiency. Theoretical analysis and simulation results show that the proposed method can effectively detect the islanding in the presence of dilution effect.
\end{abstract}

\section{Introduction}

For grid-connection inverter based distributed generation (DG) system, an important problem is the design of anti-islanding protection. The detection methods of islanding are divided into two categories: passive and active methods. At present, the research of DG system anti-islanding is mainly focused on the detection algorithm and parameter optimization of single inverter islanding detection .With the application of large capacity DG system, there are often many inverters connected to the DG system, and the research on the technology of multi grid-connection inverters islanding detection is getting more and more attention ${ }^{[1,2]}$.

Among the multi grid-connected inverters islanding detection techniques, Sandia Frequency Shift (SFS) method is widely used because of simple operation and small nondetection zone (NDZ). SFS method is based on injecting disturbance signal into the current signal of the inverter, so that the voltage frequency of point of common couple (PCC) shifts continuously after the grid is disconnected, and the islanding will be detected when the frequency exceeds the detection threshold. SFS method has good detecting effect for single grid-connection inverter; but under the condition of multi grid-connection inverters, its detecting performance significantly decreased due to the frequency disturbance of each inverter can not be synchronized, so the disturbance interfere with each other, which produce the dilution effect of islanding detection, and the islanding detection failure significantly increased. In view of this shortcoming of SFS method, this paper presents a new multi grid-connected inverters islanding detection method which is based on phase locked loop (PLL). The proposed method is a composite islanding detection method which combines SFS and Sandia Voltage Shift (SVS) method, and the simulation results verify the effectiveness of the proposed method ${ }^{[3]}$.

\section{Research on PLL based islanding detection technology of multi grid-connected inverters}

Principle of PLL based multi grid-connected inverters islanding detection. In order to avoid the failure of multi grid-connected inverters islanding detection, the SFS method can be used in conjunction with other islanding detection method to improve the detecting success rate. By applying disturbance to output current amplitude of inverter, the SVS method can make the voltage amplitude of PCC increased or decreased continuously after the main grid is disconnected, and the islanding is detected when the voltage amplitude of PCC is more than the detection threshold. Similar to SFS, the SVS method is also used to promote the continuous change of signal by using the positive feedback mechanism, But the voltage disturbance generated by SVS method has adverse effect on the maximum 
power tracking of DG system, which reduces the energy utilization efficiency. So the SVS method hasn't been used wildly. If the above two methods are used together to form a composite detection method, it can improve the success rate of multi grid-connected inverters islanding detection, and can avoid the adverse effect of SVS method on the energy utilization efficiency. the principle of the composite islanding detection technology is proposed as follows:

(1) SFS method is put into use after inverters connect to the grid, as the main islanding detection means, through frequency shifting to make anti-islanding protection act quickly after the main grid is disconnected.

(2) As an auxiliary islanding detection means, SVS method usually does not put into use, only when certain condition is met can SVS method be started. In the situation that SFS method failed to effectively detect the islanding due to dilution effect, SVS method is used to improve islanding detection success rate through the voltage amplitude disturbance.

(3) In order to avoid the false activation of SVS method due to load fluctuation and other reasons, if five consecutive voltage cycle the SVS start conditions are all met, then delay 0.3 seconds, and the voltage disturbance can be implement if the SVS start condition is still met after 0.3 seconds delay.

Design of virtual two-phase PLL based on the second-order generalized integrator. In this paper, SVS method conditional start according to the frequency and amplitude variation of PCC voltage. So the frequency and amplitude variation detection is the key. Taking into account the adverse effect of harmonic interference on the detection accuracy, this paper uses a PLL which has frequency and amplitude detection function to detect the PCC voltage, here a second-order generalized integrator (SOGI) based virtual two-phase PLL is selected because it has certain filtering function. The control structure of the PLL is shown in Fig. $1^{[4]}$.

In this Fig. 1, the structure in the dotted line frame is a quadrature signal generator with SOGI (SOGI-QSG), wherein the SOGI is behind the signal $k \varepsilon$. In this paper, the PLL uses SOGI-QSG to convert signal from single free degree to two free degrees so as to realize the coordinate transformation, meanwhile, the SOGI-QSG also has filtering function, which can eliminate the harmonic component of the original signal. From Fig. 1, you can obtain the transfer function of SOGI - QSG output signal v' and qv' relative to the input signal $v$ respectively. They are

$$
\begin{gathered}
D(s)=\frac{v^{\prime}(s)}{v(s)}=\frac{k \omega s}{s^{2}+k \omega s+\omega^{2}} \\
Q(s)=\frac{q v^{\prime}(s)}{v(s)}=\frac{k \omega^{2}}{s^{2}+k \omega s+\omega^{2}}
\end{gathered}
$$

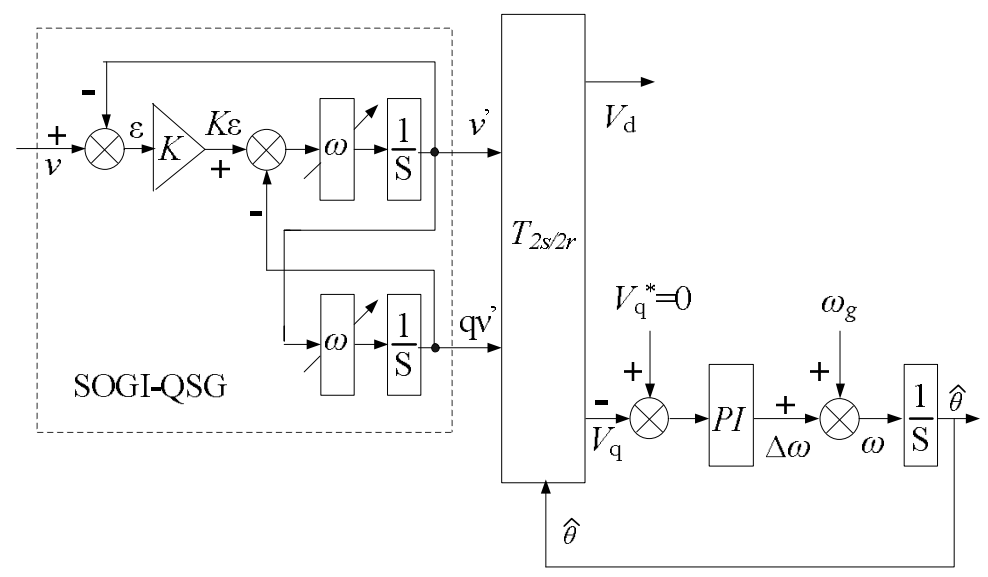

Fig. 1 Control structure of Virtual two-phase PLL based on SOGI

Starting conditions and algorithm parameters setting of SVS method. When the grid is stable, the frequency and amplitude of PCC voltage are maintained near the rated value. In the moment when 
the grid is disconnected, as long as the DG supplied power and the load power is not completely matched, the frequency and amplitude of PCC voltage will change. Therefore, the starting conditions for SVS method are

$$
\left|f-f_{\mathrm{g}}\right|>\lambda_{1} \text { or }\left|U-U_{\mathrm{g}}\right|>\lambda_{2}
$$

Where $f$ and $U$ are the detected actual values of frequency and amplitude of PCC voltage respectively, $f_{\mathrm{g}}$ and $U_{\mathrm{g}}$ are the rated value of frequency and amplitude of grid voltage, $\lambda_{1}$ and $\lambda_{2}$ are the frequency and amplitude changes which are used to start SVS islanding detection. According to the actual frequency deviation of grid voltage is usually in the range of $0.05 \mathrm{~Hz}, \lambda_{1}$ is set to $0.05 \mathrm{~Hz}$; and the deviation of voltage amplitude is limited to $7 \%$ of the rated voltage according to the national standard, considering that the amplitude of grid rated voltage is of $220 \times \sqrt{2}=311 \mathrm{~V}, \lambda_{2}$ is set to $21 \mathrm{~V}$.

For SVS method, the disturbance adds to inverter output current to make the amplitude of PCC voltage change, then the specified value of inverter output current amplitude is as follows.

$$
I_{M}^{*}=I_{M}+K_{\mathrm{V}} \cdot \Delta U
$$

Where $I_{\mathrm{M}}{ }^{*}$ and $I_{\mathrm{M}}$ are the specified value and the detected value of the inverter output current amplitude, $K_{\mathrm{V}}$ is the voltage positive feedback coefficient of SVS algorithm, $\Delta U$ is the amplitude difference between PCC voltage of last cycle and grid rated voltage. In order to ensure the effective implementation of SVS method, the voltage positive feedback coefficient $K_{\mathrm{V}}$ must be set correctly. $K_{\mathrm{V}}$ can be determined by the stability criterion of the control structure of voltage positive feedback.

Voltage positive feedback is realized by controlling the inverter output current amplitude, so according to (4), the control structure of voltage positive feedback is shown in Fig. 2. In this figure, $\mid Z$ $(j \omega) \mid$ is the circuit impedance at PCC, After the grid disconnection, $|\mathrm{Z}(\mathrm{j} \omega)|$ is the impedance of local load, $U(k T)$ is the amplitude of PCC voltage, $U_{\mathrm{g}}$ is the rated voltage of grid, $I_{\mathrm{M}}(k T)$ is the output current amplitude of inverter, and $K_{\mathrm{V}}$ is the positive feedback coefficient of voltage.

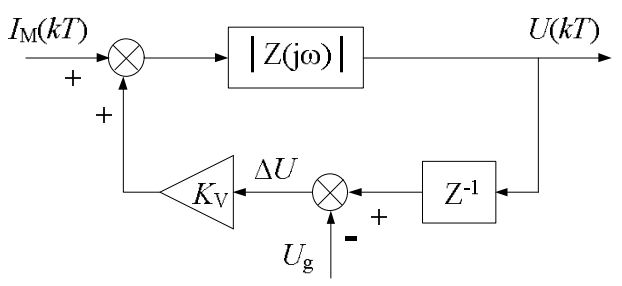

Fig. 2 structure diagram of voltage feedback control

The following expression can be get from Fig. 2

$$
U(Z)=\frac{I_{\mathrm{M}}(Z)-k_{\mathrm{v}} \cdot U_{\mathrm{g}}}{|Z(j \omega)|^{-1}-k_{\mathrm{v}} \cdot z^{-1}}
$$

Its characteristic equation is

$$
|Z(j \omega)|^{-1}-k_{\mathrm{v}} \cdot z^{-1}=0 \text { or } \quad z=k_{\mathrm{v}} \cdot Z(j \omega) \mid
$$

By $Z$ domain stability criterion of control system, the control structure is unstable when $|Z(j \omega)|>1$, then from (6) it can be obtained: 


$$
K_{\mathrm{v}}>\frac{1}{|Z(j \omega)|}
$$

As long as $K_{\mathrm{V}}$ meets (7), the control structure in Fig. 2 will be in an unstable state after the grid disconnection, so that the amplitude of PCC voltage continued shifting until beyond the over/under voltage threshold to detect the islanding ${ }^{[5]}$.

\section{Simulation results}

To verify the effectiveness of the proposed PLL based multi grid-connected inverters islanding detection method, the Matlab simulation is carried out. The simulation model is composed of two single phase grid-connected inverters to constitute the DG system, as shown in Fig. 3. System configuration parameters are as follows: the grid is $220 \mathrm{~V} / 50 \mathrm{~Hz}$ single-phase AC; Two inverters take unity power factor current control strategy, their output power are all $500 \mathrm{~W}$ and match the power of local parallel RLC load, load quality factor $Q_{\mathrm{f}}=2.5$. The algorithm parameters of islanding detection are: SFS method $c f_{0}=0.05, K=0.07$ and SVS method $K_{\mathrm{V}}=0.06$. The dilution effect of islanding detection is often caused by the inverter frequency detection error. So set inverter1 without frequency detection error, thus the SFS frequency disturbances shift frequency upward after the grid disconnection; while inverter 2 was set to have $-0.2 \mathrm{~Hz}$ frequency detection error, therefore the SFS frequency disturbances in inverter 2 shift frequency downward after the grid disconnection, and the dilution effect of islanding detection will occur after the main grid is disconnected.

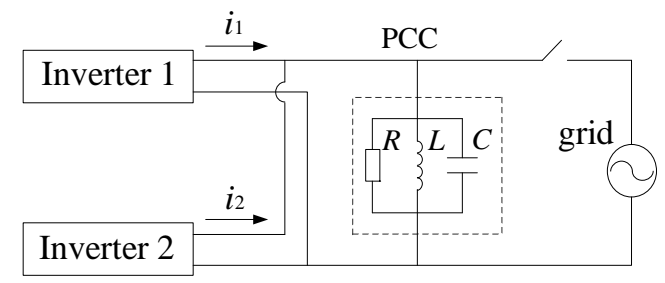

Fig. 3 DG system with two sets of grid connected inverters

Fig. 4(a) is the islanding detection simulation waveform when the SFS method is used alone. From top to bottom the signals in Fig. 4(a) are the grid current signal, the output current signal of inverter1, the output current signal of inverter 2 and the voltage signal of PCC. As can be seen from Fig. 4(a), at time of 0.1 seconds after the grid is disconnected, because of the dilution effect, the two inverters frequency disturbances offset each other, the frequency shift is slow so it hasn't reached the detecting threshold at the time of 2 second. According to the requirements of national standard, the DG system should detect the generation of islanding within 2 seconds, Fig. 4(a) shows that the islanding detection is failure ${ }^{[6]}$.

Fig. 4(b) shows the islanding detection simulation test in which the proposed PLL based Composite islanding detection method is adopted. In Fig. 4(b), from top to bottom the 4 signals are the same as those in Fig. 4(a). After the grid disconnection SFS method can make a certain frequency shift, so even if the power between DG supply and load is completely matched, the starting condition of SVS method is still relatively easy to meet. After the start of the SVS method, the voltage positive feedback disturbance is implemented, which makes the PCC voltage change continuously, once the voltage amplitude reaches the over/under voltage threshold the islanding will be detected. In Fig. 4(b), you can see that the grid is disconnected at time of 0.1 seconds, and the inverter output currents and PCC voltage are reduced to 0 at about 0.59 seconds, indicating that the islanding has been successfully detected and the islanding protection has been implemented, the detecting time is $0.59-0.1=0.49$ seconds, which can meet the requirements of the national standard for islanding detection time. 


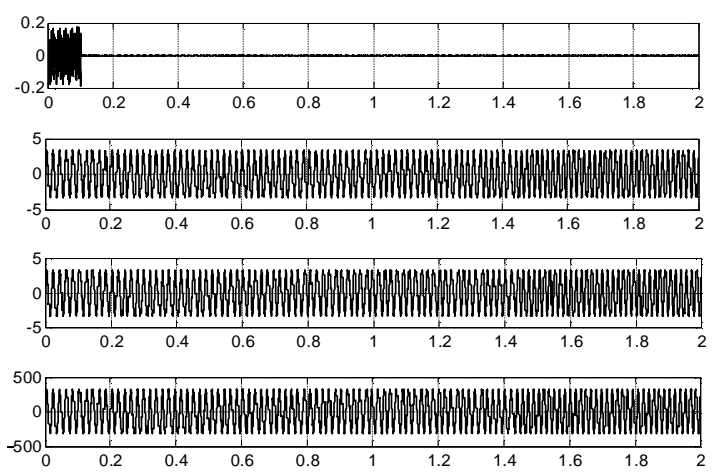

(a)
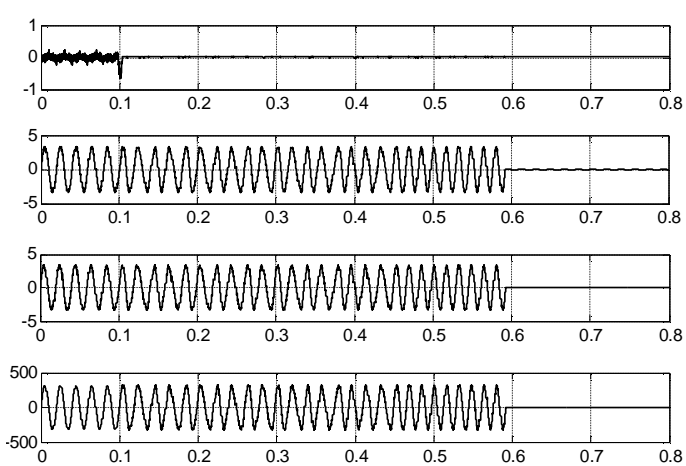

(b)

Fig. 4islanding detection simulation waveform, (a) adopting SFS method alone, (b)adopting PLL based Composite islanding detection method

\section{Conclusion}

Under the condition of multi grid-connection inverters, SFS method may have the dilution effect problem, which can lead to the islanding detection failure. This paper proposes a composite multi grid-connected inverters islanding detection method, the new method combines SFS and SVS method, in the case that SFS method can not effectively detect the islanding, conditional start SVS method to improve the success rate of islanding detection. Because SVS method does not implement during grid normal state, the continued voltage disturbance is avoided so that the energy efficiency of DG system hasn't been affected. For the proposed method, the detection of frequency and amplitude variation is the key to Correctly start SVS method, in this paper a PLL is used to overcome the harmonics caused detection error. Theoretical analysis and simulation results show that, compared with SFS method is used alone, the proposed method can effectively compensate for the lack of SFS method, so that the islanding detection performance for multi grid-connection inverters improved significantly.

\section{Acknowledgements}

Sponsored by the university natural science research project of Anhui Province (KJ2015A245), and Fund of Tongling University (2014tlxyrc03)

\section{References}

[1] Xiaolong Chen, Yongli Li, IEEE Transaction on power electronics. 29 (2014) 4672-4683.

[2] E Serban, C Pondiche, M Ordonez, IEEE Transaction on power electronics. 30 (2015) 3106-3121.

[3] L.A.C. Lopes, Yongzheng Z, IEEE Transactions on Power Delivery. 23 (2008) 480-486.

[4] P Rodriguez, R Teodorescu, I Candela, A.V Timbus, M Liserre, F Blaabjerg, IEEE power electronics specialists conference. Jeju, South Korea, 2006, pp.1-7

[5] V.John, Y. Zhihong, A Kolwalkar, IEEE Transactions on Power Electronics. 19(2004) 1177-1183.

[6] S. Wang, H. Yang, L. Wang, GB/T 19939-2005 technical requirements of grid-connected photovoltaic system, national standard of the people's Republic of China, Nov, 2005. 